\title{
The Dynamics of a Spherical Robot of Combined Type by Periodic Control Actions
}

\author{
Yu. L. Karavaev, A. A. Kilin
}

This paper presents the results of the study of the dynamics of a real spherical robot of combined type in the case of control using small periodic oscillations. The spherical robot is set in motion by controlled change of the position of the center of mass and by generating variable gyrostatic momentum. We demonstrate how to use small periodic controls for stabilization of the spherical robot during motion. The results of numerical simulation are obtained for various initial conditions and control parameters that ensure a change in the position of the center of mass and a variation of gyrostatic momentum. The problem of the motion of a spherical robot of combined type on a surface that performs flat periodic oscillations is also considered. The results of numerical simulation are obtained for different initial conditions, control actions and parameters of oscillations.

Keywords: spherical robot, nonholonomic constraint, small periodic control actions, stabilization

Received May 20, 2019

Accepted July 12, 2019

The work of Yu. L. Karavaev (Sections 3, 4) was supported by the Russian Science Foundation under grant 18-71-00096. The work of A. A. Kilin (Sections 1, 2) was supported by the Russian Science Foundation under grant 19-71-30012 and performed in Steklov Mathematical Institute of Russian Academy of Sciences.

Yury L. Karavaev

karavaev_yury@istu.ru

Center for Technologies in Robotics and Mechatronics Components, Innopolis University

ul. Universitetskaya 1, Innopolis, 420500 Russia

M. T. Kalashnikov Izhevsk State Technical University

ul. Studencheskaya 7, Izhevsk, 426069 Russia

Alexander A. Kilin

aka@rcd.ru

V. A. Steklov Mathematical Institute RAS

ul. Gubkina 8, Moscow, 119991 Russia 


\section{Introduction}

In recent studies of nonholonomic systems the problems of moving a Chaplygin sleigh and Chaplygin top by small periodic control actions are considered. The results confirm the possibility of constant acceleration (speedup) of the wheeled vehicle due to the periodic change in the mass distribution [1,2], as well as acceleration of the Chaplygin top with the help of an internal rotor [3]. In this case, the acceleration mechanism is called Fermi acceleration and is observed in systems with two and a half or more degrees of freedom.

A large number of works are devoted to pendulum systems with a vibrating point of suspension $[4-8]$ in which the dynamics of systems and the conditions for their stability are investigated. The results obtained in these studies can be used to create more complex systems, including mobile robots. For example, spherical robots have been actively developed recently. A detailed overview of existing designs of spherical robots can be found in the dissertation by Ylikorpi [9] and in $[10-14]$.

This paper presents the results of the study of the dynamics of a real spherical robot of combined type in the case of control using small periodic oscillations. The spherical robot is set in motion by controlled change of the position of the center of mass and by generating variable gyrostatic momentum [15-17].

We demonstrate how to use small periodic controls for stabilization of the spherical robot during motion. The results of numerical simulation are obtained for various initial conditions and control parameters that ensure a change in the position of the center of mass and a variation of gyrostatic momentum.

The problem of the motion of a spherical robot of combined type on a surface that performs flat periodic oscillations is also considered. The results of numerical simulation are obtained for different initial conditions, control actions and parameters of oscillations. Possible modes of motion of the spherical robot on the oscillating plane are discussed.

\section{Equations of motion of a spherical robot of combined type}

Consider a spherical robot of combined type rolling without slipping on a horizontal absolutely rough plane (Fig. 1). To describe the dynamics of the spherical robot, we define two coordinate systems: a fixed (inertial) coordinate system $O \boldsymbol{\alpha} \boldsymbol{\beta} \boldsymbol{\gamma}$ with unit vectors $\boldsymbol{\alpha}, \boldsymbol{\beta}, \boldsymbol{\gamma}$, and a moving coordinate system $C \boldsymbol{e}_{1} \boldsymbol{e}_{2} \boldsymbol{e}_{3}$ with unit vectors $\boldsymbol{e}_{1}, \boldsymbol{e}_{2}, \boldsymbol{e}_{3}$, attached to the pendulum so that the unit vector $\boldsymbol{e}_{1}$ is perpendicular to the plane of rotation of the pendulum and the unit vector $\boldsymbol{e}_{3}$ is directed along its symmetry axis.

This scheme can be implemented with various designs in practice. For example, by modification of the famous pendulum mechanism installing the rotor on it. But the simplest design is realized in the case of installation of the wheeled platform with rotor inside the spherical shell. Figure 1b shows a three-dimensional model of the proposed design of a spherical robot of combined type. In this figure, a wheeled platform moves inside the spherical shell - 1 along the rim -2 . The platform is driven by two wheels -5 with individual drive motors -6 , synchronized by the control system. The profile of the drive wheels, as well as the material from which they are made, ensure that they roll without slipping around the rim. The contact of the driving wheels with the rim is provided by a spring-loaded roller -7 . A rotor -3 actuated by an motor with a gearbox - 4, ensures the rotation of the spherical robot relative to the vertical axis. 


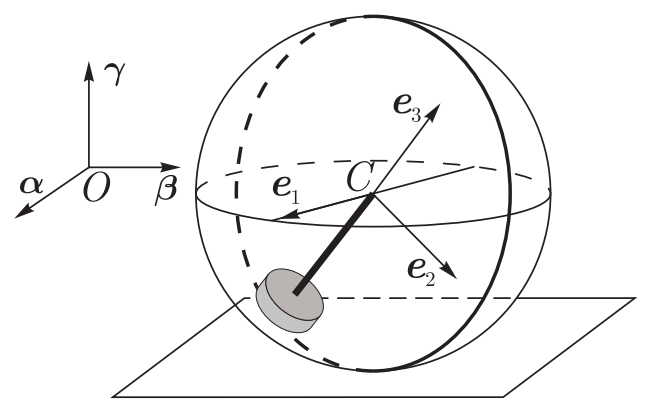

(a)

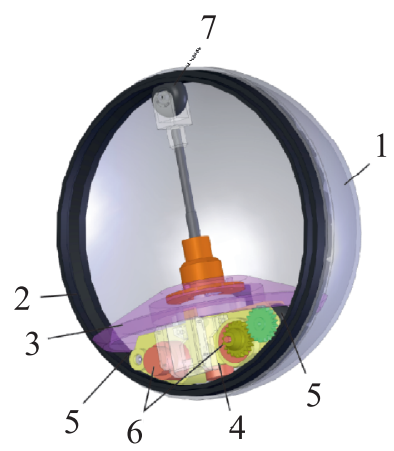

(b)

Fig. 1. A schematic model of a spherical robot of combined type (a) and a 3D model of prototype (b).

The origin of the moving coordinate system is at the geometric center of the shell $C$ (see Fig. 1). In what follows, all vectors are referred to the moving coordinate system $C_{e_{1}} \boldsymbol{e}_{2} \boldsymbol{e}_{3}$. The position of the system is given by the coordinates of the center of the sphere $\boldsymbol{R}=(x, y, 0)$ in the fixed reference frame, by the angle of rotation $\vartheta$ of the pendulum about the axis $\boldsymbol{e}_{1}$, by the angle of rotation $\varphi$ of the rotor relative to the axis $\boldsymbol{e}_{3}$ and by the orthogonal matrix of rotation $\mathbf{Q}$ of the moving coordinate system relative to the fixed one. The columns of the matrix $\mathbf{Q}$ are the coordinates of the fixed vectors $\boldsymbol{\alpha}, \boldsymbol{\beta}, \boldsymbol{\gamma}$, referred to the moving coordinate system $C_{\boldsymbol{e}_{1}} \boldsymbol{e}_{2} \boldsymbol{e}_{3}$ : $\mathrm{Q}=(\boldsymbol{\alpha}, \boldsymbol{\beta}, \boldsymbol{\gamma})$.

The absence of slipping at the point of contact of the shell with the plane is described by the nonholonomic constraint

$$
\boldsymbol{F}=\boldsymbol{v}-R_{s} \Omega \times \gamma=0
$$

where $\boldsymbol{v}$ and $\boldsymbol{\Omega}$ are the velocity of the center and the angular velocity of rotation of the shell, respectively, $R_{s}$ is the radius of the shell, and $\gamma=\left(\gamma_{1}, \gamma_{2}, \gamma_{3}\right)^{T}$ is the unit vector of the fixed reference frame in the direction of the vertical axis.

The kinetic and the potential energy of the sphere-pendulum system can be represented in the following form:

$$
T=\frac{1}{2} m_{s} \boldsymbol{v}^{2}+\frac{1}{2}\left(\boldsymbol{\Omega}, \mathbf{I}_{s} \boldsymbol{\Omega}\right)+\frac{1}{2} m_{b} \boldsymbol{v}_{b}^{2}+\frac{1}{2}\left(\boldsymbol{\omega}, \mathbf{I}_{b c} \boldsymbol{\omega}\right), \quad U=-m_{b} R_{b} \mathrm{~g}\left(\boldsymbol{\gamma}, \boldsymbol{e}_{3}\right),
$$

where $m_{s}$ and $\mathbf{I}_{s}=\operatorname{diag}\left(I_{s}, I_{s}, I_{s}\right)$ are the mass and the central tensor of inertia of the spherical shell, $m_{b}$ and $\mathbf{I}_{b c}=\operatorname{diag}\left(I_{b c 1}, I_{b c 1}, I_{b c 3}\right)$ are the mass and the central tensor of inertia of the pendulum, respectively, and the velocity of the center of mass of the pendulum $\boldsymbol{v}_{b}$ and its angular velocity $\boldsymbol{\omega}$ are given by

$$
v_{b}=\boldsymbol{v}-R_{b} \omega \times e_{3}, \quad \boldsymbol{\omega}=\Omega+e_{1} \dot{\vartheta}+e_{3} \dot{\varphi}
$$

where $R_{b}$ is the distance from the center of the sphere to the center of mass of the pendulum.

The equations governing the dynamics of the system considered can be written in the form of the d'Alembert-Lagrange equations of the second kind in quasi-velocities with undetermined multipliers and forcing actions. As shown in [15], the equations of motion for the 
variables $\dot{\varphi}, \dot{\vartheta}, \Omega, \gamma$ decouple from the complete system and take the following form:

$$
\begin{aligned}
& \left(\boldsymbol{e}_{\mathbf{3}}, \mathbf{I}_{b}\left(\dot{\boldsymbol{\Omega}}+\ddot{\varphi} \boldsymbol{e}_{\mathbf{3}}\right)\right)=K_{\varphi}, \\
& \left(\boldsymbol{e}_{\mathbf{1}}, \mathbf{I}_{b}\left(\dot{\boldsymbol{\Omega}}+\ddot{\vartheta} \boldsymbol{e}_{\mathbf{1}}\right)+\boldsymbol{\Omega} \times\left(\left(\mathbf{I}_{s}+\mathbf{I}_{b}\right) \boldsymbol{\Omega}+\dot{\varphi} \mathbf{I}_{b} \boldsymbol{e}_{\mathbf{3}}\right)-\right. \\
& \left.\quad-m_{b} R_{b} R_{s} \boldsymbol{e}_{\mathbf{3}} \times\left(\left(\dot{\boldsymbol{\Omega}}+\dot{\vartheta} \boldsymbol{e}_{\mathbf{1}} \times \boldsymbol{\Omega}\right) \times \gamma\right)+m_{b} R_{b} \mathrm{~g} \boldsymbol{\gamma} \times \boldsymbol{e}_{\mathbf{3}}\right)=K_{\vartheta}, \\
& \quad\left(\mathbf{I}_{s}+\mathbf{I}_{b}\right) \dot{\boldsymbol{\Omega}}+\ddot{\vartheta} \mathbf{I}_{b} \boldsymbol{e}_{\mathbf{1}}+\ddot{\varphi} \mathbf{I}_{b} \boldsymbol{e}_{\mathbf{3}}+\left(\boldsymbol{\Omega}+\dot{\vartheta} \boldsymbol{e}_{\mathbf{1}}\right) \times\left(\left(\mathbf{I}_{s}+\mathbf{I}_{b}\right) \boldsymbol{\Omega}+\dot{\vartheta} \mathbf{I}_{b} \boldsymbol{e}_{\mathbf{1}}+\dot{\varphi} \mathbf{I}_{b} \boldsymbol{e}_{\mathbf{3}}\right)+ \\
& +\left(m_{s}+m_{b}\right) R_{s}^{2} \boldsymbol{\gamma} \times\left(\left(\dot{\boldsymbol{\Omega}}+\dot{\vartheta} \boldsymbol{e}_{\mathbf{1}} \times \boldsymbol{\Omega}\right) \times \boldsymbol{\gamma}\right)-m_{b} R_{b} R_{s}\left(\boldsymbol{e}_{\mathbf{3}} \times\left(\left(\dot{\boldsymbol{\Omega}}+\dot{\vartheta} \boldsymbol{e}_{\mathbf{1}} \times \boldsymbol{\Omega}\right) \times \boldsymbol{\gamma}\right)+\right. \\
& \left.+\boldsymbol{\gamma} \times\left(\left(\dot{\boldsymbol{\Omega}}+\ddot{\vartheta} \boldsymbol{e}_{\mathbf{1}}\right) \times \boldsymbol{e}_{\mathbf{3}}+\left(\boldsymbol{\Omega}+\dot{\vartheta} \boldsymbol{e}_{\mathbf{1}}\right) \times\left(\left(\boldsymbol{\Omega}+\dot{\vartheta} \boldsymbol{e}_{\mathbf{1}}\right) \times \boldsymbol{e}_{\mathbf{3}}\right)\right)\right)+m \mathrm{~g} R_{b} \boldsymbol{\gamma} \times \boldsymbol{e}_{\mathbf{3}}=0 \\
& \dot{\gamma}=\boldsymbol{\gamma} \times\left(\boldsymbol{\Omega}+\dot{\vartheta} \boldsymbol{e}_{\mathbf{1}}\right),
\end{aligned}
$$

where $\mathbf{I}_{b}=\operatorname{diag}\left(I_{b 1}, I_{b 1}, I_{b 3}\right)=\operatorname{diag}\left(I_{b c 1}+m_{b} R_{b}^{2}, I_{b c 1}+m_{b} R_{b}^{2}, I_{b c 3}\right)$ is the tensor of inertia of the pendulum relative to the center of the sphere, $K_{\theta}$ is the moment of external forces (control action) which defines the rotation of the pendulum in the given plane, and $K_{\varphi}$ is the moment of external forces which defines the rotation of the rotor about its symmetry axis (the rod of the pendulum). Supplementing Eqs. (2.1) with the kinematic relations describing the motion of the center of the spherical robot and the rotation of the moving axes relative to the fixed axes

$$
\dot{\mathbf{Q}}=\widetilde{\Omega} \mathbf{Q}+\dot{\theta} \mathbf{A Q}, \quad \dot{\boldsymbol{r}}=\mathbf{Q}^{\top} \boldsymbol{v},
$$

where the matrices $\widetilde{\boldsymbol{\Omega}}$ and $\mathbf{A}$ have the form

$$
\widetilde{\boldsymbol{\Omega}}=\left(\begin{array}{ccc}
0 & \Omega_{3} & -\Omega_{2} \\
-\Omega_{3} & 0 & \Omega_{1} \\
\Omega_{2} & -\Omega_{1} & 0
\end{array}\right), \quad \mathbf{A}=\left(\begin{array}{ccc}
0 & 0 & 0 \\
0 & 0 & 1 \\
0 & -1 & 0
\end{array}\right),
$$

we obtain a closed system of equations that completely describes the rolling of the spherical robot on the plane.

The control of a spherical robot of combined type with a feedback stabilizing the given partial solutions of the free system is considered in $[18,19]$. In the approach considered, the feedback depends on the current position and the velocities of the spherical robot, does not depend on the specific type of trajectory, and involves specifying the control torques $K_{\vartheta}$ and $K_{\varphi}$.

The results of simulation of the motion of the spherical robot of combined type using feedback, as presented in $[18,19]$, confirm the possibility of stabilizing the spherical robot. However, the question of choosing and verifying the values of the coefficients $\widetilde{k}_{\vartheta}$ and $\widetilde{k}_{\varphi}$ remains open. The results of experimental investigations of the proposed algorithm show that the control actions for stabilization are similar to periodical oscillations [16, 17].

\section{Motion in the case of periodically oscillating control actions}

Consider the influence of control actions in the form of periodic oscillation on the stability of motion.

We choose the smooth acceleration from the initial rest position of the pendulum and the motion along the straight line as the base motion. It is realized by the deviation of the pendulum 
in the form

$$
\dot{\theta}(t)=\left\{\begin{array}{l}
0, \quad t<0.055 \\
0.314(\sin (10.0 \pi t-0.55 \pi))^{2}, \quad 0.055<t \leqslant 0.105, \\
0.314, \quad t>0.105
\end{array}\right.
$$

Periodic control actions are provided by periodic rotations of the rotor of the pendulum, which are specified as

$$
\dot{\varphi}(t)=\left\{\begin{array}{l}
0, \quad t<0.1 \\
0.05(\sin (10.0 \pi t))^{3}, \quad t \geqslant 0.1
\end{array}\right.
$$

The corresponding dependences $\dot{\varphi}(t)$ and $\dot{\theta}(t)$ are shown in Fig. 2.

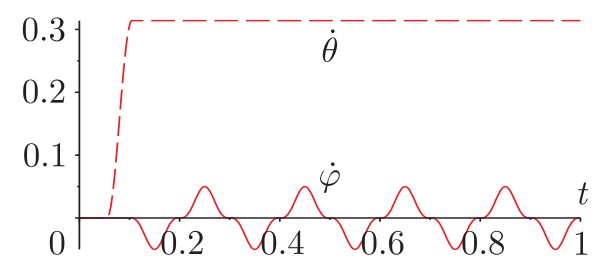

Fig. 2. Graphical representation of control actions for the spherical robot.

Depending on the initial conditions $\mathbf{Q}(0)$, the spherical robot will perform various oscillations in the process of movement. Consider the most illustrative examples for different initial conditions, which we define as follows:

$$
\begin{aligned}
& I C_{1}: \boldsymbol{\alpha}=(1,0,0), \boldsymbol{\beta}=(0,1,0), \boldsymbol{\gamma}=(0,0,1) \\
& I C_{2}: \boldsymbol{\alpha}=(1,0,0), \boldsymbol{\beta}=(0,0.7,-0.714), \boldsymbol{\gamma}=(0,-0.714,0.7) \\
& I C_{3}: \boldsymbol{\alpha}=(0.7,0,0.714), \boldsymbol{\beta}=(0,1,0), \boldsymbol{\gamma}=(-0.714,0,0.7) .
\end{aligned}
$$

Figure 3 shows the results of numerical simulation of the system (2.1) with regard to (3.1) and (3.2) and various initial conditions for the mass-geometric parameters of a real spherical robot: $R_{s}=0.150 \mathrm{~m}, m_{s}=1.625 \mathrm{~kg}, \mathbf{I}_{s}=\operatorname{diag}\left(25.27 \cdot 10^{-3}, 20.73 \cdot 10^{-3}, 25.27 \cdot 10^{-3}\right) \mathrm{kg} \cdot \mathrm{m}^{2}$, $R_{r}=0.087 \mathrm{~m}, m_{r}=2.46 \mathrm{~kg}, I_{r}=5.64 \cdot 10^{-3} \mathrm{~kg} \cdot \mathrm{m}^{2}, m_{b}=3.25 \mathrm{~kg}, \mathbf{I}_{b}=\operatorname{diag}\left(31.88 \cdot 10^{-3}\right.$, $\left.30.59 \cdot 10^{-3}, 8.76 \cdot 10^{-3}\right) \mathrm{kg} \cdot \mathrm{m}^{2}$.

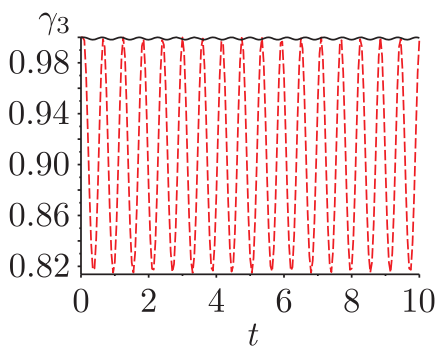

(a)

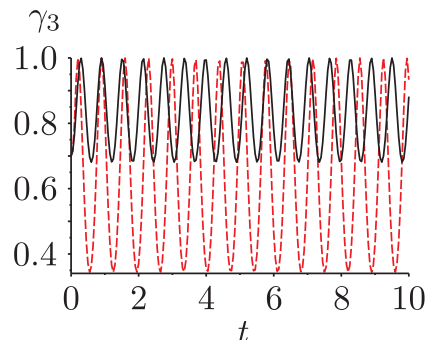

(b)

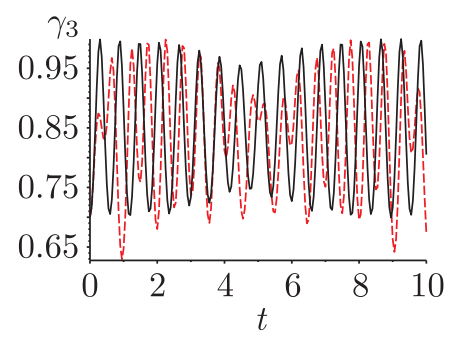

(c)

Fig. 3. Oscillations of a spherical robot during its motion by control actions (3.1) and initial conditions: a) $I C_{1}$, b) $I C_{2}$, c) $I C_{3}$. The evolution of the component $\gamma_{3}$ without periodic control action $(\dot{\varphi}=0)$ is shown as dashed line, and the solid line corresponds to periodic control action (3.2).

The results obtained and presented in Fig. 3 show that, regardless of the initial conditions, small periodic oscillations contribute to the stabilization of the motion of a spherical robot, while, for example, the constant rotation of the rotor leads to significant deviations of the trajectory [16]. In practice, such small periodic oscillations of control actions can be used to stabilize 
motion in the case of deviations caused by obstacles, deviations of geometric dimensions, and uncertainties.

\section{Dynamics of the spherical robot rolling on a periodically oscillating underlying surface}

Consider the problem of motion of the spherical robot on a periodically oscillating underlying surface (Fig. 4). In this section, we choose as a spherical robot a rotor model with a displaced (but fixed) center of mass, which is set in motion by changing the intrinsic gyrostatic momentum. Oscillations of the surface are realized by the angular velocity of the plane $\boldsymbol{\Omega}(t)$.

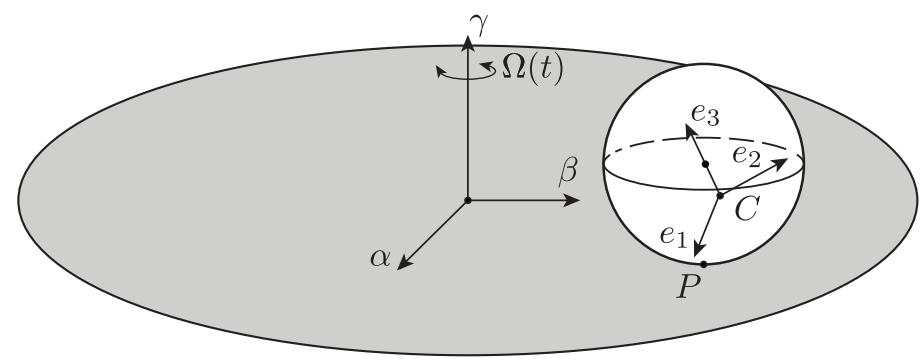

Fig. 4. The model of a spherical robot rolling on an oscillating plane.

The nonholonomic constraint in the case of a rotating underlying plane is

$$
\boldsymbol{f}=\boldsymbol{v}+\boldsymbol{\omega} \times \boldsymbol{r}-\Omega(t) \boldsymbol{\gamma} \times \boldsymbol{R}=0, \quad \boldsymbol{R}=x \boldsymbol{\alpha}+y \boldsymbol{\beta},
$$

where $\boldsymbol{r}$ is the radius vector of the contact point from the center of mass position, $\boldsymbol{R}$ is the radius vector of the ball center in the moving axes.

Following [20], one can obtain equations of motion for the system of interest. After reduction the system under consideration takes the form in variables $\boldsymbol{\omega}, \boldsymbol{\gamma}, \boldsymbol{R}$ :

$$
\begin{gathered}
\widetilde{\mathbf{I}}=(\widetilde{\mathbf{I}} \boldsymbol{\omega}+\boldsymbol{K}) \times \boldsymbol{\omega}+m \boldsymbol{r} \times(\dot{\boldsymbol{r}} \times \boldsymbol{\omega})+m \Omega(t) \boldsymbol{r} \times(\dot{\boldsymbol{R}} \times \boldsymbol{\gamma})-m \Omega(t)(\boldsymbol{\omega}, \boldsymbol{\gamma}) \boldsymbol{R} \times \boldsymbol{r}+ \\
+m \dot{\Omega}(t) \boldsymbol{r} \times(\boldsymbol{R} \times \boldsymbol{\gamma})+m g \boldsymbol{\gamma} \times \boldsymbol{r}, \\
\dot{\boldsymbol{\gamma}}=\boldsymbol{\gamma} \times \boldsymbol{\omega}, \quad \dot{\boldsymbol{R}}=\boldsymbol{R} \times(\boldsymbol{\omega}-\Omega(t) \boldsymbol{\gamma})+\boldsymbol{r} \times \boldsymbol{\omega}-(\boldsymbol{\omega}, \boldsymbol{c} \times \boldsymbol{\gamma}) \boldsymbol{\gamma},
\end{gathered}
$$

where $\boldsymbol{K}=\left(K_{1}, K_{2}, K_{3}\right)$ is the gyrostatic moment, $\boldsymbol{c}=\left(c_{1}, c_{2}, c_{3}\right)$ is the constant displacement of the center of mass relative to the geometrical center of the sphere, and the radius vector $\boldsymbol{r}$ is expressed in terms of $c$ and $\gamma$ as follows:

$$
r=-R_{s} \gamma-c .
$$

Consider the results of numerical simulation of the system (4.2) for various control actions given by the gyrostatic moment: without any control $(\boldsymbol{K}=0)$, with constant gyrostatic moment $(\boldsymbol{K}=$ const $)$ and by periodical gyrostatic moment. All results of simulations are presented for angular velocity $\Omega(t)=\sin (t)$.

The results presented in Fig. 5 show that, in the case of oscillations of the underlying surface, stabilization by means of small periodic control actions is inefficient. 


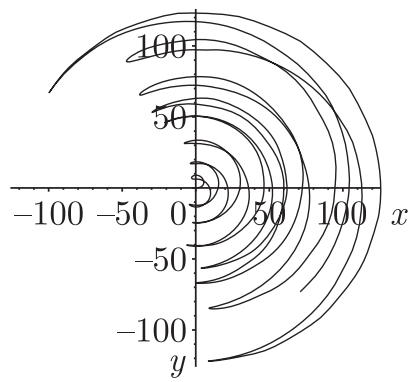

(a)

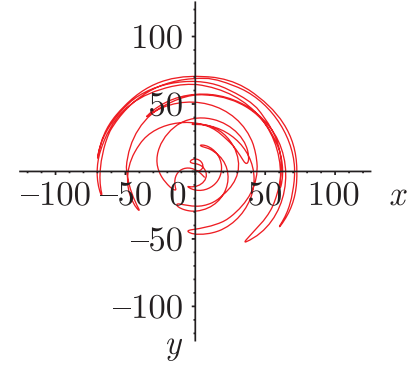

(b)

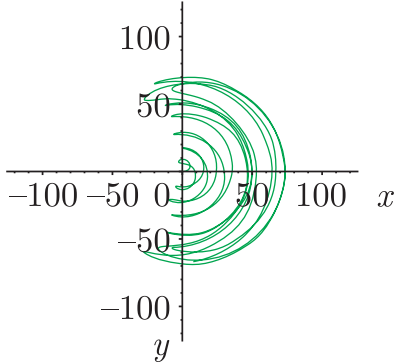

(c)

Fig. 5. Trajectories of the spherical robot moving on an oscillating plane in the case of: a) $\boldsymbol{K}(\boldsymbol{t})=0$, b) $\boldsymbol{K}(\boldsymbol{t})=(0,0,1)$, c) $\boldsymbol{K}=(0,0,0.5 \cdot \sin (2 \cdot t))$.

\section{Conclusions}

In conclusion, we present the most important problems obtained in this work and plans for the future in the study of the rolling of a spherical robot in the case of small periodic control actions or on the surface performing small oscillations:

1. Small periodic oscillations contribute to the stabilization of the motion of a spherical robot keeping the desired direction of motion of the robot.

2. In addition to oscillations of the underlying surface in the horizontal plane, oscillations along the vertical should also be considered. Taking them into account will allow modeling more complex dynamic behavior.

3. Future research will be aimed at developing an algorithm for calculation of control actions to compensate for the influence of the oscillating underlying surface.

\section{Acknowledgments}

The authors thank I. A. Bizyaev and I. S. Mamaev for fruitful discussions of the results obtained.

\section{References}

[1] Bizyaev, I. A., Borisov, A. V., and Kuznetsov, S. P., Chaplygin Sleigh with Periodically Oscillating Internal Mass, Europhys. Lett., 2017, vol. 119, no. 6, 60008, 7 pp.

[2] Bizyaev, I. A., Borisov, A.V., and Mamaev, I. S., The Chaplygin Sleigh with Parametric Excitation: Chaotic Dynamics and Nonholonomic Acceleration, Regul. Chaotic Dyn., 2017, vol.22, no.8, pp. 955-975.

[3] Borisov, A. V., Kilin, A. A., and Pivovarova, E. N., Speedup of the Chaplygin Top by Means of Rotors, Dokl. Phys., 2019, vol.64, no. 3, pp. 120-124; see also: Dokl. Akad. Nauk, 2019, vol. 485, no.3, pp. 285-289.

[4] Belichenko, M.V., On the Stability of Pendulum-type Motions in the Approximate Problem of Dynamics of a Lagrange Top with a Vibrating Suspension Point, Russian Journal of Nonlinear Dynamics, 2018, vol. 14, no. 2, pp. 243-263.

[5] Bardin, B. S. and Savin, A. A., On the Orbital Stability of Pendulum-Like Oscillations and Rotations of a Symmetric Rigid Body with a Fixed Point, Regul. Chaotic Dyn., 2012, vol. 17, nos. 3-4, pp. 243-257. 
[6] Yudovich, V.I., Vibrodynamics and Vibrogeometry in Mechanical Systems with Constraints, Uspekhi Mekh., 2006, vol.4, no. 3, pp. 26-158 (Russian).

[7] Markeyev, A.P., The Equations of the Approximate Theory of the Motion of a Rigid Body with a Vibrating Suspension Point, J. Appl. Math. Mech., 2011, vol.75, no. 2, pp. 132-139.

[8] Kholostova, O. V., On the Periodic Motion of Lagrange's Top with Vibrating Suspension, Mech. Solids, 2002, no. 1, pp. 26-38.

[9] Ylikorpi, T., Mobility and Motion Modelling of Pendulum-Driven Ball Decoupled Models Robots: for Steering and Obstacle Crossing, Doctoral Dissertations, School of Electrical Engineering, 2017, $251 \mathrm{pp}$.

[10] Chase, R. and Pandya, A., A Review of Active Mechanical Driving Principles of Spherical Robots, Robotics, 2012, vol. 1, no. 1, pp. 3-23.

[11] Chen, W.-H., Chen, C.-P., Yu, W.-S., Lin, C.-H., and Lin, P.-C., Design and Implementation of an Omnidirectional Spherical Robot Omnicron, in Proc. 2012 IEEE/ASME Int. Conf. on Advanced Intelligent Mechatronics, Kaohsiung (Taiwan), 2012, pp. 719-724.

[12] Crossley, V.A., A Literature Review on the Design of Spherical Rolling Robots, Preprint (Carnegie Mellon Univ., Pittsburgh, PA, 2006).

[13] Akella, P., O'Reilly, O., and Sreenath, K., Controlling the Locomotion of Spherical Robots or Why BB-8 Works, J. Mechanisms Robotics, 2019, vol.11, no. 2, 024501, 4 pp.

[14] Tafrishi, S. A., Svinin, M., Esmaeilzadeh, E., and Yamamoto, M., Design, Modeling, and Motion Analysis of a Novel Fluid Actuated Spherical Rolling Robot, J. Mechanisms Robotics, 2019, vol. 11, no. 4, 041010, $10 \mathrm{pp}$

[15] Kilin, A. A., Pivovarova, E. N., and Ivanova, T. B., Spherical Robot of Combined Type: Dynamics and Control, Regul. Chaotic Dyn., 2015, vol. 20, no. 6, pp. 716-728.

[16] Kilin, A. A. and Karavaev, Yu. L., Experimental Research of Dynamic of Spherical Robot of Combined Type, Nelin. Dinam., 2015, vol.11, no.4, pp. 721-734 (Russian).

[17] Borisov, A. V., Kilin, A.A., Karavaev, Yu. L., and Klekovkin, A. V., Stabilization of the Motion of a Spherical Robot Using Feedbacks, Appl. Math. Model., 2019, vol.69, pp. 583-592.

[18] Ivanova, T. B., Kilin, A. A., and Pivovarova, E. N., Controlled Motion of a Spherical Robot with Feedback: 1, J. Dyn. Control Syst., 2018, vol. 24, no. 3, pp. 497-510.

[19] Ivanova, T. B., Kilin, A.A., and Pivovarova, E. N., Controlled Motion of a Spherical Robot with Feedback: 2, J. Dyn. Control Syst., 2019, vol. 25, no. 1, pp.1-16.

[20] Borisov, A. V., Mamaev, I.S., and Bizyaev, I. A., The Jacobi Integral in Nonholonomic Mechanics, Regul. Chaotic Dyn., 2015, vol.20, no.3, pp. 383-400. 